\title{
Timing in Neurogenerative Disorders of the Basal Ganglia
}

\author{
Deborah L. Harrington ${ }^{* * *}$ and Stephen M. Rao ${ }^{\dagger}$
}

1

\section{Introduction}

Time is an integral facet of behavior that structures our perceptions, experiences, and memories. Although there is no clinical disorder that is specifically characterized by timing disturbances, some are known to alter certain interrelated cognitive functions, such as planning. A host of neurological and psychiatric diseases that affect basal ganglia, cerebellar, and cerebral cortex functioning affect timing within the scale of tenths of milliseconds on up to seconds or even minutes. As such, there has been a lively debate over the neural sources of temporal processing deficits in various disease processes. Presently, there is substantial support for the centrality of the striatum and dopamine neurotransmission in explicit timing (Balci et al., 2012; Hohn et al., 2011; Lake and Meck, 2012; Meck, 2006a,b; Rammsayer, 1993). This chapter discusses timing disturbances in two disorders of the basal ganglia in which dopaminergic functioning is decreased, namely Parkinson's disease (PD) and prodromal Huntington disease (HD). There is a growing interest in these disorders since temporal processing deficits contribute to the breakdown in the spatiotemporal organization of movement in PD (Vercruysse et al., 2012) and timing ability is a marker of proximity to a diagnosis of manifest HD (Harrington et al., 2012; Rowe et al., 2010). This chapter places an emphasis on functional imaging investigations of timing disturbances in PD and prodromal $\mathrm{HD}$, as this has received little attention in the literature to date. To set the stage for our discussion, we first review two influential timing models that underscore the crucial roles of key component processes and neurophysiological mechanisms. The framework provided by these models, together with advancements in neuroanatomical connectivity, has guided research into the

* va San Diego Healthcare System, San Diego, CA, USA.

** Department of Radiology, University of California, San Diego, La Jolla, CA, USA.

$\dagger$ Schey Center for Cognitive Neuroimaging, Neurological Institute, Cleveland Clinic, Cleveland, он, USA.

(C) DEBORAH L. HARRINGTON \& STEPHEN M. RAO, 2015 | DOI 10.1163/9789004230699_009 
neurocognitive mechanisms of timing and its breakdown. We then give an overview of timing disturbances in PD and the effects of dopamine therapy, followed by research into prodromal phases of HD. This section is not intended to provide a thorough treatment of this topic as there are several excellent, recent reviews of temporal processing disturbances in PD (Allman and Meck, 2012; Koch, Oliveri, and Caltagirone, 2009). We then discuss emerging functional imaging research, which is beginning to reveal brain circuits that govern timing deficits in these disorders. Lastly, we consider the clinical relevance of temporal processing dysfunction in these diseases and future avenues for research.

\section{$2 \quad$ Models of Timing}

We lead with a brief description of two influential models that have driven research in neurologically intact adults and in clinical disorders of temporal processing. The information processing model has provided a strong framework for studying component processes of interval timing, which can have distinct effects on timing accuracy and variability. In contrast, a leading neurophysiological model delineates the physiological and neuroanatomical properties of timing networks, thereby fostering an understanding of the functional significance of abnormalities in corticostriatal networks that underlie timing disturbances.

\subsection{Information Processing Model}

Over the last two decades there has been an explosion of research into the neuroanatomical underpinnings of interval timing in disorders of the basal ganglia. Initially, a driving force behind much of this work was scalar expectancy theory (SET; Gibbon, 1977; Gibbon, Church, and Meck, 1984), which defined sources of timing variability that were derived from clock, memory, and decision processes (Figure 8.1A). Empirical findings in pharmacological and lesion studies in animals further suggested that the clock process depended on dopamine neurotransmission and the striatum (Buhusi and Meck, 2002; Maricq and Church, 1983; Meck, 1996). In SET, timing is implemented via a pacemaker mechanism, which represents time through the accumulation of pulses. Pulses are turned on and off by a mode switch and then passed onto an accumulator to be counted. Perceived duration is intimately related to the interplay between the pacemaker and the level of attention given to the passage of time. Attention affects the mode switch, which controls the flexible starting and stopping of pulses from the pacemaker, thereby enabling 
anticipation of predictable events. Attention also affects the accumulation of pulses, such that when attention is diverted there is a shortening of perceived duration, suggesting that pulses are lost. The current representation of time is stored in working memory, where it is maintained in accord with current goals and over time, more enduring interval representations are stored in long-term memory. A decision process compares pulse counts from the accumulator with ones in memory to determine when or how to respond. At this stage, strategic or attentional factors can influence decision processes by biasing response thresholds that determine when the current time is sufficiently close to the remembered time. Altogether, the perception of time emerges from the interplay among these component processes. For this reason, SET continues to be an influential framework for unraveling the potential influence of various cognitive processes on disturbances in temporal processing. Potential sources of timing deficits are inferred through different patterns of accuracy and variability, which has been discussed in detail by others (Allman and Meck, 2012; Wearden, 1999).

\subsection{Neurophysiological Model}

As knowledge grew about the neurobiological bases of timing, the challenge of unraveling the functional significance of temporal processing deficits in neurological disorders became more apparent. This challenge can be better appreciated upon consideration of the complexity of corticostriatal connectivity (Figure 8.1B). Cortical networks subserving different behaviors have separate, partially segregated pathways to the striatum that return to the same areas via direct and indirect pathways. There is also a hyper-direct pathway from the cortex to the subthalamic nucleus (STN), which circumvents the striatum. Dopaminergic input to the striatum and cerebral cortex is via the substantia nigra pars compacta (SNc; nigrostriatal pathway) and the ventral tegmental area (VTA; mesolimbic and mesocortical pathways), respectively. The cerebellum also has separate parallel connections with the cerebral cortex (Akkal, Dum, and Strick, 2007; Clower, Dum, and Strick, 2005). Moreover, the cerebellum and the basal ganglia communicate with each other by way of output nuclei from each region (dentate nuclei) and the sTN (Figure 8.1C; Bostan, Dum, and Strick, 2010; Hoshi et al., 2005). Thus, timing might emerge from complex interactions within corticostriatal, cortico-cortical, and/or striatalcerebellar networks.

The Striatal Beat Frequency Model (SBF; Matell and Meck, 2004), which evolved from an elegant body of work in rodents, captured the inherent interactive nature of timing networks (Matell, Meck, and Nicolelis, 2003; Matell et al., 2011; Meck et al., 2012). By this model (Figure 8.1B), the striatum receives 


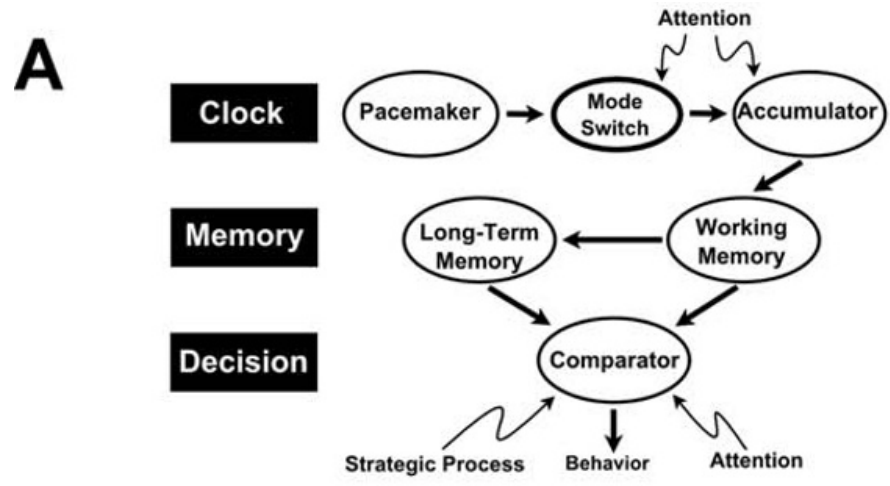

B

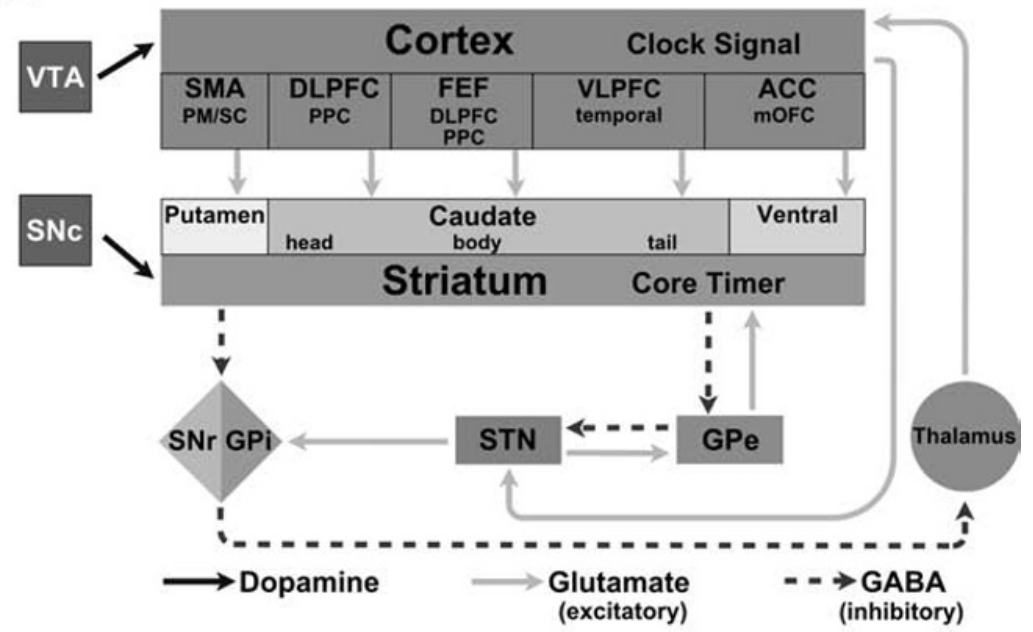

C

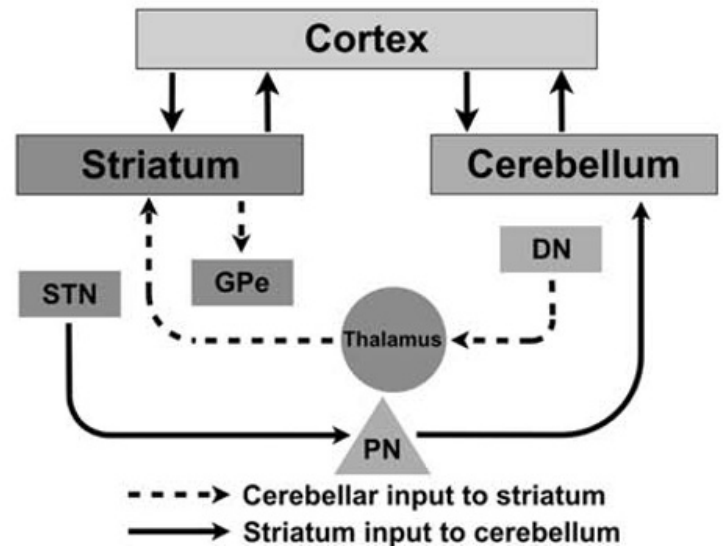

FIGURE 8.1 Interval timing models and brain networks. A: Illustration of the three component processes of an information processing model of timing, scalar expectancy theory 
cortical oscillatory activity, which evolves as a function of event duration, thereby serving as a clock signal. The striatum detects the state of the cortical oscillatory patterns and responds when a pattern matches previously reinforced or intrinsically important states, thereby serving as a core timer. Tonic dopamine and glutamate are thought to alter cortical oscillation frequencies, whereas nigrostriatal phasic dopamine signals the onset and the offset of a timed event. Dopamine also strengthens striatal synapses that are activated by the frequency pattern of cortical neurons to a timed event. So by the SBF, timing depends on dopamine input from different pathways and corticostriatal interactions. An implication of the SBF is that timing disturbances in basal ganglia disorders should arise from changes in pathways involving the striatum, but also other brain circuits that govern cognitive functions. This is supported by psychopharmacological challenges in rodents that demonstrate that cognitive enhancers (atomoxetine and physostigmine) and disruptors (scopolamine and chlordiazepoxide) respectively improve and worsen temporal precision in the peak procedure task (Balci et al., 2008). Though not specifically addressed by the SBF, diminished striatal functioning might also engage brain

FIGURE 8.1 (CONT.) (SET). The clock component consists of a pacemaker that represents time through the accumulation of pulses. Pulses are turned on and off by $a$ switch and then passed into an accumulator to be counted. Accumulated pulses are encoded into working memory and over time, more enduring interval representations are stored in long-term memory. Decision processes compare pulse counts from the accumulator with ones in memory to determine when or how to respond. B: The neuroanatomically connectivity of the striatum and cortex is illustrated in the context of the Striatal Beat Frequency Model ( $S B F$ ). By this model, the striatum receives cortical oscillatory activity, which evolves as a function of event duration, thereby serving as a clock signal. The striatum detects the state of the cortical oscillatory patterns and responds when a pattern matches previously reinforced or intrinsically important states, thereby serving as a core timer. Tonic dopamine and glutamate alter cortical oscillation frequencies; nigrostriatal phasic dopamine signals the onset and the offset of a timed event. Dopamine also strengthens striatal synapses that are activated by the frequency pattern of cortical neurons to a timed event. C: Illustration of anatomical pathways that enable two-way communication between the striatum and cerebellum. Acc = anterior cingulate cortex; $D L P F C=$ dorsolateral prefrontal cortex; $D N=$ dentate nucleus; $F E F=$ frontal eye fields; $G P e=$ globus pallidus externa; $G P i=$ globus pallidus interna; $P P C=$ posterior parietal cortex; $P M=$ premotor; $P N=$ pontine nucleus; $S M A=$ supplementary motor area; $s c=$ sensory cortex; $S N c=$ substantia nigra pars compacta; $S N r=$ substantia nigra pars reticulata; $S T N=$ subthalamic nucleus; $V L P F C=$ ventrolateral prefrontal cortex; VTA = ventral tegmental area. 
circuits that compensate via degeneracy mechanisms, wherein different anatomical networks are engaged (Merchant, Harrington, and Meck, 2013). Degeneracy in timing systems is plausible since time-related cell activity is not only found in the basal ganglia, but also the cerebellum, thalamus, posterior parietal cortex, prefrontal cortex, and the supplementary motor area (SMA and preSMA; Merchant et al., 2013). Timing in different behavioral contexts is also associated with different neural architectures. For example, implicit and explicit timing are thought to respectively depend on the cerebellum and striatum (Coull, Cheng, and Meck, 2011). Similarly, cortico-cortical systems are more engaged when timed movements that are externally paced, whereas the striatum is engaged when movements are self-paced or internally timed (Rao et al., 1997; Taniwaki et al., 2003). Thus, different neural systems may be capable of timing. At the same time, it is far from clear whether degeneracy mechanisms actually compensate (i.e., improve performance) for diminished timing. We will return to this issue when discussing neuroimaging studies of timing in PD and prodromal HD.

\section{Temporal Processing Deficits in Basal Ganglia Disorders}

Temporal processing in PD and prodromal HD is of keen interest due to the centrality of the striatum and dopamine neurotransmission in timing. Though traditionally considered disorders of movement, timing disturbances in both diseases are not simply due to motor symptoms, since they are found in contexts that minimize motor output. Temporal processing has been far more studied in PD than prodromal HD. In this section, behavioral studies will be reviewed in PD, followed by those in prodromal HD. For each disorder, we first briefly review the underlying neuropathology. Then clinical features are discussed that are relevant to elucidating the neurobehavioral mechanisms of temporal processing deficits and interpreting discrepancies that have been reported across studies.

\subsection{Parkinson's Disease}

3.1.1 Neuropathology and Symptoms

The principal neuropathological finding in PD is dopamine cell loss in nigrostriatal pathway and to lesser extent, the mesocortical/limbic pathway. PD not only presents with classic motor symptoms (tremor, rigidity, slowness, postural instability, freezing), but it has a progressive impact on cognition. In PD without dementia, cognitive decline is often subtle and attributed to frontostriatal 
dysfunction, as it most commonly affects executive functions, attention, and working memory. By SET, some of these functions may interact with the clock, thereby complicating the interpretation of the source for timing deficits. It is also important to recognize that there is considerable clinical heterogeneity of cognitive symptoms in PD (Carbon, Edwards, and Eidelberg, 2003; Lewis et al., 2003; Williams-Gray et al., 2009). This heterogeneity likely relates to individual differences in pathological changes and their severity, although this is not well understood nor is the effect of dopamine therapy on various cognitive functions. Another important consideration is that there are day-to-day fluctuations in symptoms and individual differences in responsiveness to medication therapy. Thus, a gamut of clinical phenotypes may underlie some discrepancies reported across studies with regard to whether timing is impaired in PD or affected by dopamine therapy.

\section{1 .2 \\ Temporal Processing in Parkinson's Disease and the Effect of Dopamine Therapy}

Most studies have reported temporal processing deficits in PD across a variety of tasks including time production or reproduction (Elsinger et al., 2003; Harrington, Haaland, and Hermanowicz, 1998; Jones et al., 2011; Jones et al., 2008; Koch et al., 2008; O'Boyle, Freeman, and Cody, 1996; Pastor et al., 1992a; Pastor et al., 1992b; Perbal et al., 2005; Wing, Keele, and Margolin, 1984), time estimation (Koch et al., 2004a, 2004b; Malapani, Deweer, and Gibbon, 2002; Pastor et al., 1992b; Smith et al., 2007; Wild-Wall et al., 2008), and time discrimination (Harrington et al., 2011b; Harrington , Haaland, and Hermanowicz, 1998; Rammsayer and Classen, 1997; Riesen and Schnider, 2001). Basal ganglia lesions also disrupt the detection and production of intervals (Schwartze et al., 2011). However, the mechanisms underlying these deficits are unclear. By SET, a slowdown in the clock process in PD should alter timing accuracy, thereby producing an underestimation of time. Although this has been reported for time estimation (Malapani et al., 2002; Pastor et al., 1992; Smith et al., 2007; WildWall et al., 2008), measures of accuracy that are straightforward to interpret within SET are often not available for other tasks. For example, repetitively timed movements in PD can be over produced, especially for subsecond intervals (Elsinger et al., 2003; Harrington, Haaland, and Hermanowicz, 1998; Jones et al., 2011; Pastor et al., 1992a), but this may not be due to a timing disturbance per se. Rather, the finding may relate to a clinical feature of PD, namely motor festination, wherein individuals attempt to correct for the decreased amplitude of repetitive movements by speeding them up (e.g., gait, handwriting). Most studies of timing reproduction or production also report greater timing variability in PD, which by SET may reflect changes both in the clock and other 
component processes. Though time discrimination paradigms circumvent the potentially confounding effects of motor symptoms on timing, increased temporal difference thresholds in PD (i.e., the least amount of time that can be perceived between two intervals) may also relate to changes in the clock and/ or other component processes. As an aside, several reviews suggest that the basal ganglia are more important for timing within the range of seconds, whereas the cerebellum subserves timing functions with the subsecond range (Koch et al., 2009; Lewis and Miall, 2003). Although this has received support in some studies of PD (Koch et al., 2008; Smith et al., 2007), others have found timing deficits in the range of subseconds (Elsinger et al., 2003; Harrington, Haaland, and Hermanowicz, 1998; O'Boyle et al., 1996; Rammsayer and Classen, 1997; Riesen and Schnider, 2001). The reasons for the discrepancies are not entirely clear and will require carefully conducted psychophysical studies across a range of timing tasks to sort this out. It is also important to recognize that while dichotomies can provide useful frameworks to advance research, they often oversimplify the organization of neurocognition.

As for the effect of dopamine replacement therapy, it can improve (O'Boyle et al., 1996; Pastor et al., 1992a, 1992b), have no effect (Elsinger et al., 2003; Harrington et al., 2011; Jahanshahi et al., 2010; Koch et al., 2008), or hinder timing performance (Jones et al., 2008). At first glance, the absence of an effect of dopamine on timing in PD seems at odds with the SBF model and the effects of dopamine agonists and antagonists on timing (Buhusi and Meck, 2002; Lake and Meck, 2012; Meck, 1996; Meck et al., 2012). However, dopamine replacement therapy is not complete in PD, which concurs with timing dysfunction even when patients are tested on their medication therapy (Harrington et al., 2011; Harrington, Haaland, and Hermanowicz, 1998; Rammsayer and Classen, 1997; Riesen and Schnider, 2001). There are also lingering effects of medications when they are temporarily stopped for up to 24 hours, because they are long acting and there are individual differences in treatment responsiveness. The effect of dopamine therapy on cognition is not well understood, but it can hinder performance if there is too much dopamine in functionally-intact corticostriatal circuits that support a cognitive function (Cools, 2006). Altogether, these factors can make it difficult to evaluate the influence of dopamine on temporal processing in the PD model.

An important consideration is that timing deficits have not always been found in PD. For example, normal performance was reported on a test of motor timing (Spencer and Ivry, 2005) and on several different tests of time perception (Wearden et al., 2008). The reasons for this are unknown, but may be due to the insensitivity of tasks when time discriminations are rather easy (i.e., $25 \%$ or more than the duration of anchor stimuli) and/or when feedback is regularly given (Wearden et al., 2008). In addition, timing deficits in PD 
correlate with disease severity (Artieda et al., 1992). Several studies have tested early-stage patients (Spencer and Ivry, 2005), who despite considerable dopamine cell loss may have the capacity to compensate for timing difficulties. The cerebellum may be one compensatory route (Kotz and Schwartze, 2011), possibly because it predicts and finely tunes behavioral states based on efferent copy of sensory and motor information. Cortical systems might support compensatory processing in PD as well. Apart from these issues, the finding that there are subgroups of patients who do and do not exhibit timing disturbances (Merchant et al., 2008) resonates with the substantial heterogeneity of clinical phenotypes in the disease, day-to-day fluctuations in symptoms, and individual differences in response to dopamine therapy. Neurodegenerative disorders of the basal ganglia eventually alter cortical functioning, which may be another source of interval timing disturbances. This prospect was suggested in an early report that damage to the right hemisphere of the prefrontal (dorsolateral prefrontal and premotor cortices) and the inferior parietal cortex disrupted time perception (Harrington, Haaland, and Knight, 1998). Additionally, damage to the right, but not the left hemisphere, produced elevated temporal discrimination thresholds that correlated with a decreased ability to flexibly reorient attention. This finding comports with a report that time estimation is improved in PD after repetitive transcranial magnetic stimulation (rTMS) to the right dorsolateral prefrontal cortex, but not the SMA (Koch et al., 2004b). Moreover, rTMS to the supramarginal gyrus of the right hemisphere in healthy adults causes a distortion in perceived duration (Wiener et al., 2012; Wiener et al., 2010). Thus, frontoparietal systems, which govern attention functions, interact with timekeeping processes (Lustig, Matell, and Meck, 2005). Unfortunately, systematic investigations into cortical regions that are essential for timing in humans have been hampered by difficulties in obtaining sufficient samples of patients with focal cortical damage. For this reason, functional imaging has become increasingly important for understanding brain circuits that govern disturbances in temporal processing.

\subsection{Huntington Disease}

\subsubsection{Neuropathology and Symptoms}

Huntington disease (HD) is an autosomal dominant neurodegenerative disorder caused by a polyglutamine (CAG) expansion in the IT15 gene, which leads to the production of a mutant form of the protein huntingtin. HD usually manifests in midlife, but the age of onset varies considerably and partly depends on the CAG repeat length. The mutant huntingtin protein is expressed throughout the brain, yet the medium spiny neurons of the caudate nucleus 
and to a lesser extent, the putamen, are the most vulnerable early in the disease process. Pathological changes in cortical gray- and white-matter also gradually develop as individuals approach onset of the illness (Aylward et al., 2012; Nopoulos et al., 2010). The HD diagnosis is based on the appearance of unequivocal extrapyramidal motor symptoms (e.g., chorea, oculomotor problems, dysarthria, dystonia, gait disturbance, postural instability, rigidity, bradykinesia).

There is an inverse correlation between age of onset and CAG repeat length, such that greater repeat lengths signify a stronger genetic burden and earlier onset of illness. Because this relationship accounts for only 47 to $73 \%$ of the variability in age of illness onset (Brinkman et al., 1997; Ranen et al., 1995), there has been a concerted effort to identify early markers of the disease during the prodromal phase, when potential treatments are more likely to be effective. In prodromal HD, the striatum begins to atrophy decades before diagnosis, rendering it a good model of basal ganglia dysfunction. Pathological changes are accompanied by subtle motor and psychiatric symptoms, but also cognitive decline (e.g., processing speed, executive functions, and sensoryperceptual processes; Harrington et al., 2012; Stout et al., 2011) that typically is not clinically significant until an individual approaches diagnosis. Like PD, however, subtle cognitive changes may interact with the clock process, thereby complicating the interpretation of the source for temporal processing deficits.

\subsubsection{Temporal Processing in Huntington Disease}

Interval timing in HD has been far less studied than in PD. Deficits in timed repetitive movements were first reported in manifest HD using the classic paced tapping task wherein finger movements are initially entrained to a series of isochronous tones (synchronization phase), followed by a period of tapping at the same pace without the tones (continuation phase; Freeman et al., 1996). Abnormal timing accuracy and variability was found, irrespective of whether cues (tones) were present or absent. Recent studies have replicated this finding in manifest HD using similar motor timing tasks (Bechtel et al., 2010; Thompson et al., 2010). Deficits in time estimation and discrimination have also been found in manifest HD (Beste et al., 2007), which suggests that motor disturbances cannot entirely explain impaired motor timing. Still, sources of temporal processing dysfunction remain difficult to ascertain due to the significant cognitive disturbances in manifest HD.

A better model of basal ganglia dysfunction is prodromal $\mathrm{HD}$, since motor and cognitive changes are typically subtle. In prodromal $\mathrm{HD}$, paced-tapping variability, but not accuracy, is abnormal for both subsecond and suprasecond 
intervals during synchronization and continuation phases of the task (Hinton et al., 2007; Zimbelman et al., 2007). Timing variability also increased nonlinearly with estimated years to onset, demonstrating that timing dysfunction accelerates as individuals approach a clinical diagnosis (Hinton et al., 2007). This finding was replicated for subsecond intervals by large multisite studies, which also controlled for subtle motor symptoms (Bechtel et al., 2010; Rowe et al., 2010; Stout et al., 2011). In addition, a longitudinal study of paced timing performance demonstrated that timing variability gradually increased over a two to four year period, but at a faster rate in individuals who were closer to diagnosis (Rowe et al., 2010). Thus, there is consensus that rhythmically timed movements progressively deteriorate with proximity to diagnosis.

To date, only two studies have investigated temporal processing on tasks that minimize motor output requirements. One study found time discrimination deficits (reduced accuracy) for suprasecond intervals (1200ms) in individuals close (<12 years), but not far ( $>12$ years) from diagnosis (Paulsen et al., 2004). Another study found that time discrimination was normal in prodromal HD relative to a control group (Beste et al., 2007). However, higher error rates on the task correlated with estimated proximity to diagnosis, suggesting that the absence of group differences may have been due to the small $(\mathrm{N}=12)$ heterogeneous sample of prodromal HD participants. Clearly, more studies of time perception tasks are needed to determine if temporal processing deficits in prodromal HD are independent of changes in the motor system.

Another issue is that pathological changes in the cortex (i.e., cortical thinning, white-matter atrophy, altered white-matter diffusivity) are seen more than a decade before a diagnosis of manifest PD (Dumas et al., 2012; Nopoulos et al., 2010; Paulsen et al., 2010). These changes likely cause subtle cognitive decline, which progresses with proximity to diagnosis. As in PD, cognitive dysfunction may interact with timekeeping processes, thereby rendering it difficult to identify the neurocognitive mechanisms of temporal processing dysfunction. The advent of functional imaging has therefore been a welcome development.

\section{Neuroanatomical Underpinnings of Temporal Processing Dysfunction in Basal Ganglia Disorders}

The neural underpinnings of timing dysfunction in PD and prodromal HD are not well understood due to the dearth of functioning imaging investigations. To date, there have been only four functional imaging studies of motor timing dysfunction in PD, all of which have used the paced-tapping task (Cerasa et al., 
2006; Elsinger et al., 2003; Jahanshahi et al., 2010; Yu et al., 2007). Despite differences among studies in the analysis methods and the control conditions, some results are remarkably consistent, particularly as they concern the functional role of the cerebellum in timing. However, there are also discrepancies among studies in regional patterns of timing-related dysfunction during the synchronization and the continuation phases of the task or whether PD is characterized by hypo- or hyperactivation. This may be partly a consequence of the small samples PD participants in all studies (10 or fewer), as there is considerable heterogeneity of clinical phenotypes in PD. Although the brain circuits that govern time perception deficits in PD have been far less studied (Dusek et al., 2012; Harrington et al., 2011), it is clear that patterns of regional brain dysfunction in PD depend on the extent to which timing and other cognitive processes are emphasized within a behavioral context. Another important development concerns investigations into disturbances in timing-related connectivity of the striatum (Harrington et al., 2011b; Jahanshahi et al., 2010), which appears more sensitive to the influence of dopamine therapy than conventional regional analyses of brain activation. As for prodromal HD, two studies have been conducted to date, one of timed movements (paced-tapping task; Zimbelman et al., 2007) and the other of time discrimination (Paulsen et al., 2004). Although more research is needed, timing deficits in prodromal HD appear to be governed by some similar brain circuits as in PD.

\subsection{Functional Imaging Studies of Parkinson's Disease}

\subsubsection{Timed Movements}

The neuroanatomical basis of motor timing deficits in PD was first explored by Elsinger and colleagues in an functional magnetic resonance imaging (fMRI) study of participants who were tested on (ON) and off (OFF) their medication therapy (Elsinger et al., 2003). Paced tapping (6ooms isochronous interval) was studied for both the synchronization and continuation phases. Timing was impaired in the PD group during both phases, irrespective of medication. Though the fMRI analyses did not directly compare the control and PD groups or the medication conditions, group differences were implied by two main patterns of regional activation. Regardless of medication or phase of the task, cerebellar activation was found in the control, but not the PD group, suggesting an absence of cerebellar compensation. Second, during the continuation phase, medication reinstated motor circuit activation, similar to the control group. This result suggested that internally timed movements in PD depended on the motor circuit.

Cerasa and colleagues subsequently used fMRI to study paced tapping (75oms isochronous interval) during the synchronization and continuation 
phases in PD OFF participants relative to controls (Cerasa et al., 2006). Timing was impaired in PD during both phases of the task, despite different patterns of abnormal activation in the two phases. During the synchronization phase, the PD group showed hyperactivity in the motor circuit (putamen, thalamus, SMA), the inferior frontal gyrus and insula, and the cerebellum. During the continuation phase, the PD group showed hyperactivity in the thalamus and cerebellum. Thus, in contrast to Elsinger and colleagues, externally guided rather than internally controlled timing was related to motor-circuit dysfunction. Moreover, potential compensatory responses were observed in the inferior frontal gyrus and insula (synchronization phase) and in the cerebellum (both phases).

$\mathrm{Yu}$ and colleagues partially replicated and extended these findings in an fMRI study of synchronized timing (900 and 2400ms isochronous intervals), which compared activation in PD OFF participants with controls (Yu et al., 2007). Despite no group differences in timing accuracy or variability, the PD group exhibited hypoactivation of the motor circuit, rather than hyperactivation (Cerasa et al., 2006). However, consistent with Cerasa and colleagues, hyperactivation was found in the bilateral cerebellum, but also the contralateral motor area. Importantly, ipsilateral cerebellar activation negatively correlated with contralateral putamen activation, whereas motor cortex activation correlated positive with the severity of rigidity. The authors speculated that over activation of the cerebellum might normalize timing performance, since this structure supports timed movements (Harrington et al., 2004a; Ivry and Keele, 1989). As illustrated in Figure 8.1C, this could be achieved via direct cerebellar input into the striatum (Bostan et al., 2010; Hoshi et al., 2005). The prospect that the cerebellum may support timing in PD due to degeneracy in timing systems is intriguing (Kotz and Schwartze, 2011). However, hyperactivation may also reflect a loss in the topographic specificity of basal ganglia output or generalized spreading of activity due to a loss in regional specialization (Bergman et al., 1998; Bronfeld and Bar-Gad, 2011; Pessiglione et al., 2005), which may be the result of increased neural nose (Matell and Meck, 2004).

In a positron emission tomography (РET) study, Jahanshahi and colleagues (Jahanshahi et al., 2010) also found hyperactivation of the cerebellum OFF medication during synchronization and continuation (100oms isochronous interval), despite normal timing performance, irrespective of medication. In addition, hypoactivity of frontal, temporal, and parietal regions was generally alleviated by medication, whereas hypoactivity of the caudate was not. To explore whether medication altered interactions between the caudate and the whole brain, the psychophysiological interaction (PPI) method was used to test the effective connectivity of the caudate with the whole brain. In the PPI 
analysis, the time course of a seed region (caudate) is correlated with the time courses of other brain voxels to determine if it differs as a function of medication. The main results showed that effective connectivity between the caudate and the cerebellum was stronger OFF than ON medication, which is compatible with the prospect of timing-related compensatory activation by the cerebellum (Cerasa et al., 2006; Yu et al., 2007). Interestingly, effective connectivity of the caudate with elements of the motor circuit (putamen and SMA) was also stronger OFF medication, which may relate to excessive synchronicity in corticostriatal circuits after dopamine depletion in animal models (Costa et al., 2006). In contrast, stronger connectivity of the caudate with prefrontal areas was found ON than OFF medication, possibly reflecting greater frontostriatal modulation of performance by cognitive-control networks. Altogether, the results suggest that timing in PD is associated with altered patterns of striatal connectivity with the prefrontal cortex and classic motor systems, the strength of which depends on dopamine therapy.

Although the prospect of degeneracy in timing mechanisms is intriguing, the above interpretations should be tempered by the fact that the mechanisms of effective connectivity are not well understood, nor is their relationship to regional patterns of hyper- and hypoactivity. More research is needed to determine if strengthening or weakening of connectivity in PD has a direct influence on performance or signifies dedifferentiation of activation due to reduced regional specialization. It is also unknown whether cerebellar hyperactivity or changes in striatal-cerebellar connectivity are due to disturbances in timing per se or motor-control processes. For example, the cerebellum is also hyperactive in PD during the performance of self-initiated and automatic movements (Wu and Hallett, 2005; Wu et al., 2010), presumably due to deficits in motor control. Moreover, motor symptoms in PD correlate with altered effective connectivity of the cerebellum, basal ganglia, and sMA during movement (Wu et al., 2011; Wu, Chan, and Hallett, 2010). Thus, patterns of connectivity in classic motor areas may partly relate to disturbances in motor-control processes due to the significant motor-output component of the paced-tapping task.

\subsubsection{Perceptual Timing}

With this in mind, we used fMRI to investigate brain systems that govern time perception deficits in PD and to examine the effect of dopamine therapy on neurocognition (Harrington et al., 2011b). Participants included 21 volunteers with PD and 19 older adult controls. Figure 8.2A illustrates the time discrimination task, in which a standard interval (SI) was presented, followed by a delay period and then a comparison interval (CI). The participant judged if the CI was longer or shorter in duration than the sI. Because temporal processing 
A

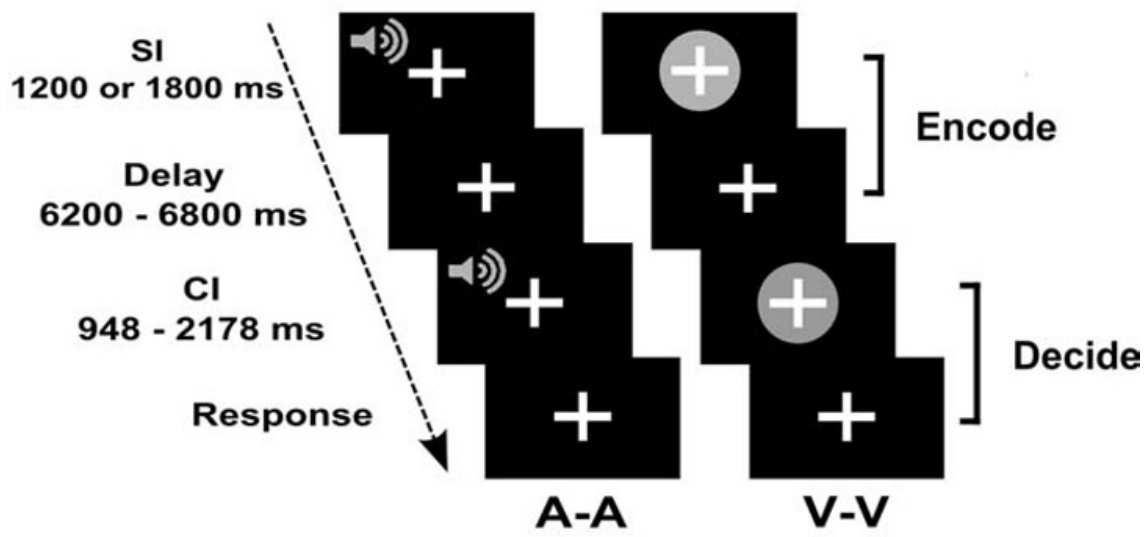

B

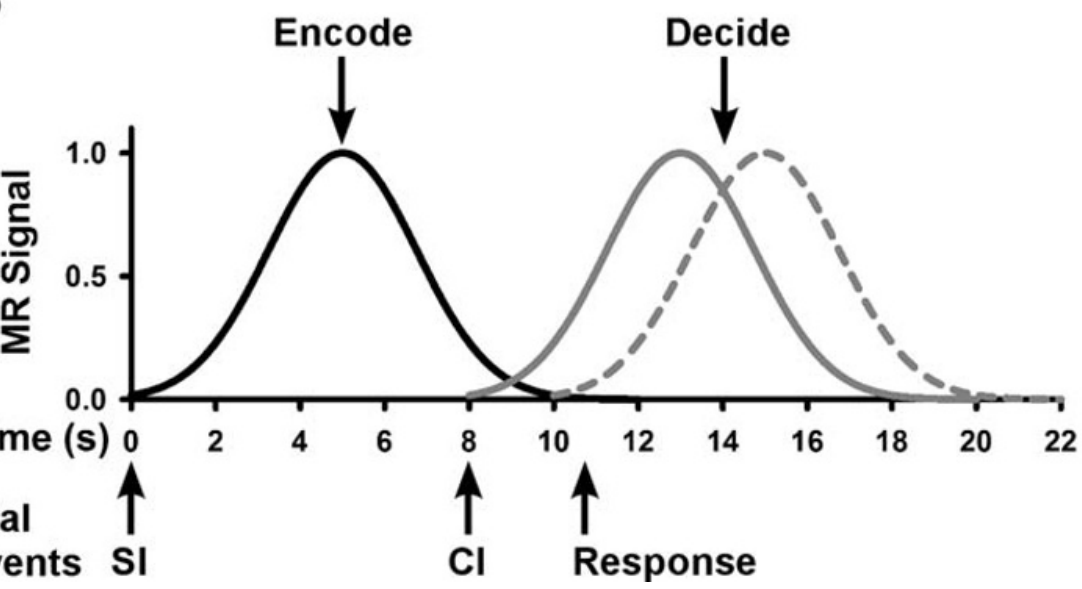

FIGURE 8.2 Time perception paradigm. A: Illustration of the trial events in the time perception task. A standard and a comparison interval were successively presented and separated by a delay. The standard (SI) was 1200 or $1800 \mathrm{~ms}$ and respectively pegged to a delay of 6800 or $6200 \mathrm{~ms}$. Three shorter and three longer comparison intervals (CI) were $\pm 7 \%$ increments of each sI. Intervals were designated by filled tones or a blue sphere. B: The three hypothetical time-course functions illustrate the expected $M R$ signal associated with encoding the standard interval (black curve), encoding the comparison interval (solid gray curve), and making a response (dotted gray curve). Arrows leading from each trial event designate their onset. The hemodynamic response peaks 4 to $6 s$ after the onset of the events. An image of the entire brain is acquired every $2 \mathrm{~s}$. The fixation cross is displayed throughout the task. Figure adapted from Harrington and colleagues (2011b). 
unavoidably engages a host of cognitive processes, we sought to deconstruct component processes by separating activation associated with encoding the SI from activation associated with encoding the CI and making a decision. This can be done by inserting a long delay period (6200 and 680oms) between the end of the SI and the onset of the CI (Harrington et al., 2004b). Figure 8.2B illustrates a hypothetical hemodynamic response function (HRF), which peaks 4 to $6 \mathrm{~s}$ after the onset of an event (e.g., SI, CI, response). By acquiring an image of the whole brain every $2 \mathrm{~s}$, the HRF for the first $12 \mathrm{~s}$ of the trial constituted brain activity associated with encoding the sI and holding it in memory (encoding phase) and the last $12 \mathrm{~s}$ of the trial constituted brain activity associated with encoding the CI and judging its duration relative to the SI (decision phase). Both phases were assumed to engage timing. We reasoned that the encoding phase would also engage working memory, whereas the decision phase would engage executive or decisional processes involved in comparing the two intervals. Activation was compared to rest (i.e., fixation plus ambient noise) to better evaluate potential dysfunction in cognitive and sensory systems that normally support timing (Bueti and Macaluso, 2011).

Time perception performance during $\mathrm{AMRI}$ was impaired in $\mathrm{PD}$, irrespective of the signal modality of intervals and dopamine therapy. In addition, volumetric analyses of anatomical MRIS revealed no significant cortical or basal ganglia atrophy in the PD group. Figure 8.3 displays areas of activation that were associated with temporal processing in both groups during the two phases of the trial. Areas color-coded blue showed normal activation in the PD OFF condition, whereas regions color-coded red showed significant group differences in activation. Despite similar patterns of regional activation during both phases of the trial, the figure shows that abnormal regional activation in PD largely depended on context-specific processes. An exception was striatal activation (caudate and putamen), which typically was hypoactive in the PD OFF condition during both phases. In the encoding period, hypoactivation was also found in the motor circuit (putamen, preSMA/sMA and cingulate, premotor cortex), an attention/working memory network (caudate, middle-frontal gyrus, inferior parietal cortex, lateral cerebellum), a memory center (parahippocampus), an attention and integration center (insula), and a sensory processing hub (vermis). Most of these regions are commonly associated with timing in young adults (Bueti and Macaluso, 2011; Coull, Nazarian, and Vidal, 2008; Coull et al., 2004; Harrington et al., 2004b; Harrington et al., 2010; Koch et al., 2004b; Melgire et al., 2005; Pouthas et al., 2005; Rao, Mayer, and Harrington, 2001; Wiener et al., 2012; Wittmann et al., 2010). In contrast, in the decision phase, we found hypoactivation in the PD OFF condition only in elements of a memory retrieval hub (posterior cingulate, parahippocampus) and in the 


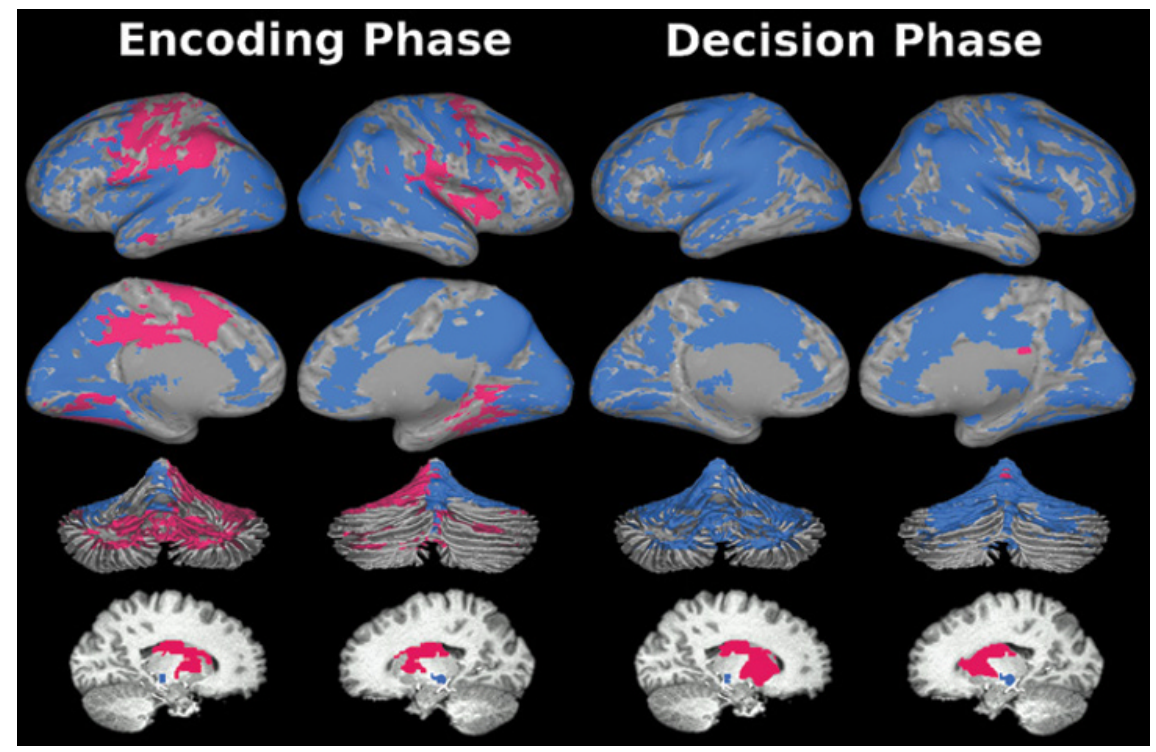

FIGURE 8.3 Regional analyses of brain activation in PD for the encoding and decision phases. Functional regions of interest (ROI) were derived by conjoining timing-related activation from the control and PD groups. Blue areas blue signify regions in which activation did not differ between the groups. Red areas showed hypoactivity in the PD OFF group relative to the control group. Brain activation is projected onto the lateral (row 1) and medial (row 2) surfaces of the left and right hemispheres, the anterior and posterior surfaces of the cerebellum (row 3 ), and the left and right basal ganglia (row 4). Brain sections are displayed in neurological view. Figure adapted from Harrington and colleagues (2011b).

vermis. These results resonate with the distinct patterns of timing dysfunction in PD that have been attributed to the storage and retrieval to temporal memories (Koch et al., 2004a; Malapani et al., 2002). Our results suggest the possibility that memory storage and retrieval are related to dysfunction in different brain circuits in PD. Interestingly, the cerebellum was hypoactive in PD OFF during both phases of the time perception task. This may suggest that cerebellar hyperactivity during motor timing (Cerasa et al., 2006; Jahanshahi et al., 2010; Yu et al., 2007) relates to motor control functions, rather than timing per se. Additional research is needed to test this proposal.

As for dopamine therapy, it had rather circumscribed effects on regional activation. In the encoding phase, it normalized activation in elements of the limbic system (insula, parahippocampus) and the vermis, but otherwise had no effect on cortical or striatal activation. In the decision phase, dopamine therapy slightly improved putamen activation and normalized vermis activity. Normalization of vermis activity during both phases may come about via the 
striatal-cerebellar pathways (Figure 8.1C), although this was not sufficient to improve timing performance.

The rather limited effect of dopamine therapy on brain activation may be related to a host of factors discussed above. Another important consideration is that conventional regional analyses of brain activation are insensitive to changes in brain networks (Rowe, 2010). Since temporal processing depends on communication of the striatum with other brain regions, it is crucial to study striatal connectivity. We therefore used the PPI method to explore whether dopamine therapy altered the effective connectivity of the striatum (caudate and putamen) with the cortex and the cerebellum. These analyses were conducted separately for activation during the encoding and decision phases of a trial. The results showed that striatal connectivity was modulated by dopamine only in the decision phase, which concurred with its effect on striatal activation during this phase only. This finding does not mean that corticostriatal connectivity is absent during the encoding phase, only that it is not altered by medication. Figure 8.4 shows that striatal connectivity was stronger OFF than ON medication with areas of the motor circuit (green), the frontoparietal attention network (purple), and the insula (blue). This effect is consistent with reports that dopamine depletion produces excessive spontaneous synchronicity in corticostriatal circuits (Costa et al., 2006; Gatev, Darbin, and Wichmann, 2006; Hammond, Bergman, and Brown, 2007), which may hinder flexible updating and integration by the striatum in contexts that call for cognitive flexibility (Lustig et al., 2005). The finding also comports with findings of stronger resting-state connectivity in PD OFF than ON medication (Baudrexel et al., 2011; Kwak et al., 2010; Stoffers et al., 2008). In contrast, left putamen connectivity was stronger ON than OFF medication with the left superior frontal gyrus (red), possibly signifying compensatory processing in some frontostriatal networks. Interestingly, the putamen and caudate did not show medication-modulated effective connectivity with the cerebellum, as the caudate does for motor timing (Jahanshahi et al., 2010).

Although it is not clear why dopamine altered striatal connectivity in the decision, but not the encoding phase, a similar result was recently reported in an fMRI study that examined regional activation during the encoding and the reproduction of intervals ranging between 5 and $11.89 \mathrm{~s}$ (Dusek et al., 2012). One clue may come from the work of Cools and colleagues (Cools, 2006) who proposed that mesocortical dopamine stabilizes cortical oscillatory activity, which in our time perception task is important during the encoding phase where interval durations must be maintained in working memory. An implication of this model is that cortico-cortico connectivity might benefit from dopamine therapy during interval encoding, which we did not explore. In contrast, 
nigrostriatal dopamine is thought to improve updating and integrative functions of basal ganglia networks, which is emphasized during the decision phase of our task. Though speculative, this model might advance an understanding of seemingly discrepant effects of dopamine therapy across different tasks, because it considers the demands placed on cognitive stability and flexibility control-processes, which respectively engage the cortex and striatum.

\subsection{Functional Imaging Studies of Huntington Disease}

\subsubsection{Timed Movements}

Since striatal atrophy is seen decades before a manifest diagnosis, it is of keen interest to delineate the brain networks that are associated with motor timing deficits in prodromal HD and to ascertain the relationship to disease burden. Structural changes in the striatum, but also the cortex, are known to correlate with motor timing proficiency. In a combined sample of prodromal HD and early manifest HD individuals, striatal atrophy and cortical thinning on the lateral and medial surfaces of the rostral-frontal and occipital-parietal cortices correlated with changes in paced-tapping accuracy to a metronome $(55 \mathrm{oms}$ isochronous interval; Bechtel et al., 2010). Though the strong association with thinning in the occipital cortex was unexpected, the finding may reflect disease progression rather than a functional association, because occipital cortex thinning begins decades before a diagnosis and progresses over time (Nopoulos et al., 2010). At the same time, structural changes are not necessarily functionally significant, since some degree of atrophy or thinning may not alter functioning.

For these reasons, functional imaging investigations are vital. To date, there has been only one functional imaging investigation into the brain circuits that govern motor timing deficits in prodromal HD (Zimbelman et al., 2007). Using the paced-tapping task (6ooms isochronous interval), we sought to identify the brain systems associated with motor timing deficits and to determine if neurocognitive dysfunction was related to disease burden. Prodromal HD participants were divided into two groups of 13 participants each based on their genetic testing (CAG repeat length) and age. One group was estimated to be further (FAR) from diagnosis (more than 12 years to onset) and the other group was closer (CLOSE) to a diagnosis (less than 12 years to estimated onset). Both groups were compared to a healthy control group. Timing variability was greater in the CLOSE, but not the FAR group during the synchronization and the continuation phases of the task. Volumetric analyses showed significant caudate atrophy in the FAR group and significant caudate and putamen atrophy in the CLOSE group. No group differences were found in cortical atrophy. 


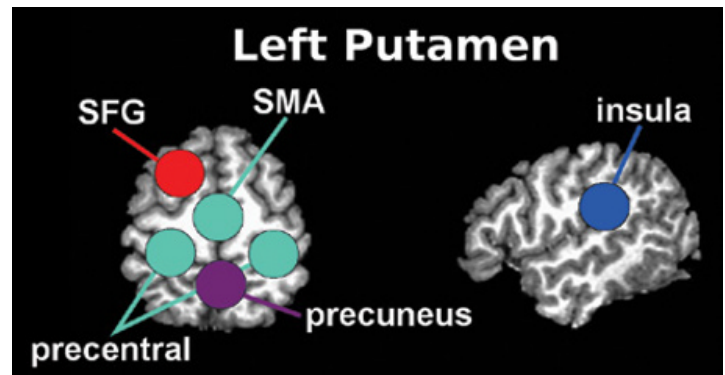

\section{Right Putamen}

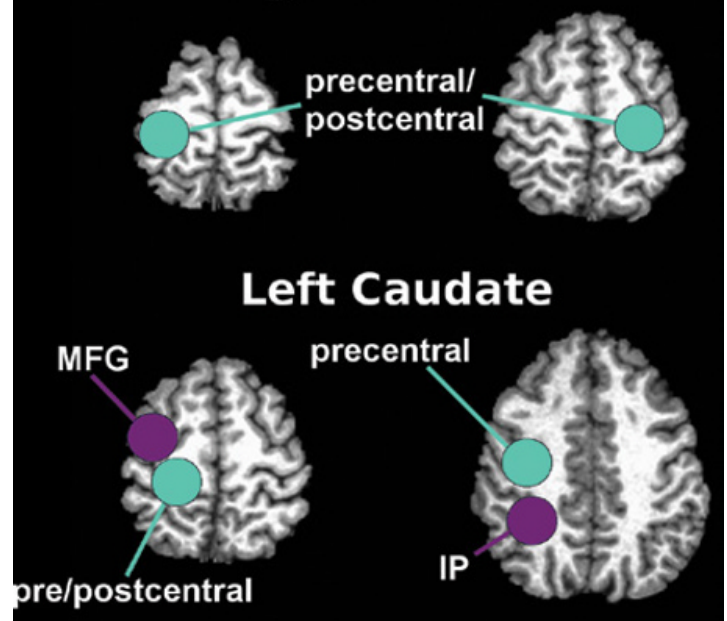

Right Caudate

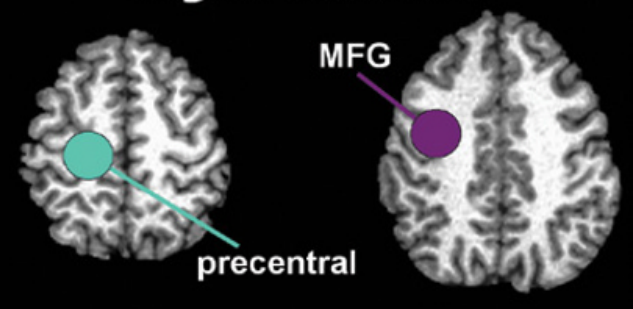

FIGURE 8.4 Cortical regions showing dopamine-modulated connectivity with the striatum during the decision phase. For each striatal seed region, effective connectivity of theputamen and caudate is illustrated on axial or sagittal brain sections, which are displayed in neurological view. Striatal connectivity was stronger OFF than ON medication with elements of the motor circuit (green areas), the frontoparietal workingmemory network (purple areas), and the limbic system (insula). Striatal connectivity was stronger ON than OFF medication with the superior frontal gyrus. IP = inferior parietal cortex; $M F G=$ middle frontal gyrus; $S F G=$ superior frontal gyrus; $S M A=$ supplementary motor area. Figure adapted from Harrington and colleagues (2011). 
For the fMRI analyses, the synchronization and continuation phases were combined and compared to a rest condition. Figure 8.5 (top) displays the regions that showed significant activation in the timing task for one or more groups. The graphs show the MR signal change in selected regions in each group. The main results revealed hypoactivation in the CLOSE group relative to the control and the FAR groups in the motor circuit (left putamen, SMA, preSMA, cingulate motor area; Figure 8.5, FAR = Controls $>$ CLOSE), the left anterior insula, and the right inferior frontal gyrus. All of these brain centers are known to exhibit timing-related activation in young adults (Bueti, van Dongen, and Walsh, 2008; Coull et al., 2008; Harrington et al., 2010). In addition, the FAR group showed two patterns of functional changes. First, there was a stepwise reduction in activation with disease burden in the right anterior cingulate and right anterior insula (Figure 8.5, Controls $>$ FAR $>$ CLOSE), suggesting early functional decline in components of the limbic system that support attention and executive functions. The FAR group also exhibited hyperactivation relative to the control and the CLOSE groups in sensory and in motor areas including the sensorimotor cortex, the precentral gyrus, medial frontal gyrus, superior temporal gyrus and the right cerebellum (Figure 8.5, FAR $>$ Controls $=$ CLOSE). This result may signify compensatory responses in early stages of neurodegeneration, similar to PD during motor timing (Jahanshahi et al., 2010; Yu et al., 2007). Hyperactivation could be an intermediate phenotype of cell dysfunction, which begins long before cell death (Tobin and Signer, 2000). Because the close group did not exhibit hyperactivation in any brain regions, this purported compensatory mechanism may weaken as the neurodegenerative process advances. Indeed, a nonlinear trajectory of activation across the continuum of cognitive impairment (i.e., increases and decreases in brain activation) has been observed for mild cognitive impairment and Alzheimer's disease (Celone et al., 2006). Nevertheless, it is also important to consider that the findings may be an early sign of dedifferentiation. To tease apart these explanations, longitudinal studies are needed that relate brain-activation patterns to timing performance.

In contrast to PD (Harrington et al., 2011b), significant caudate and/or putamen atrophy was found in the prodromal HD groups. Striatal atrophy is one of the better predictors of disease prognosis in prodromal HD (Aylward et al., 2012). Thus, one question is whether measures of cortical functioning better distinguish controls from the different prodromal HD groups, beyond other known predictors of disease prognosis including striatal atrophy, executive functioning on neuropsychological tests, and timing (accuracy and variability). We therefore performed a hierarchical discriminant analysis to examine group classification accuracy using striatal atrophy, task performance, and 


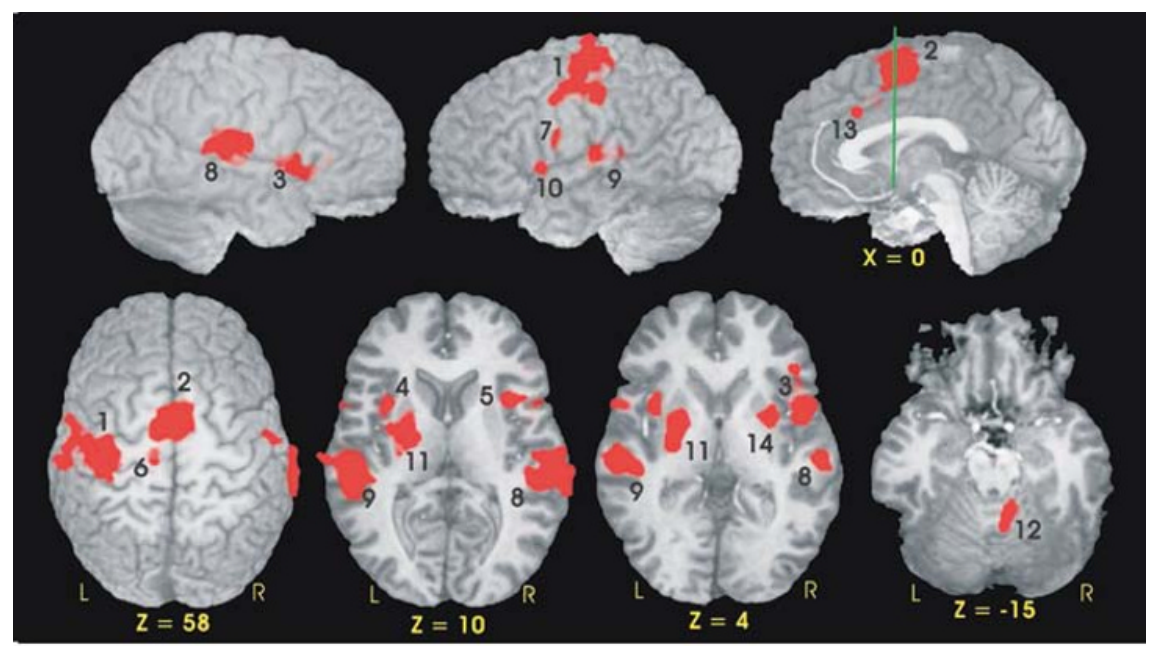

FAR $>$ Controls $=$ CLOSE

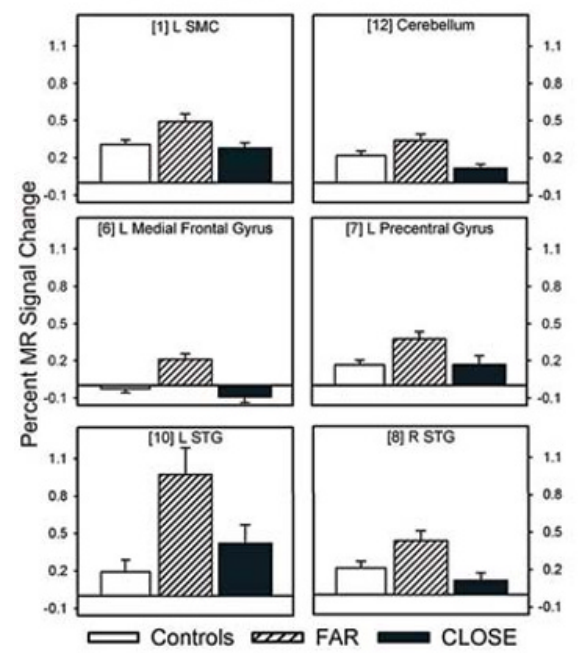

Controls $>$ FAR $>$ CLOSE

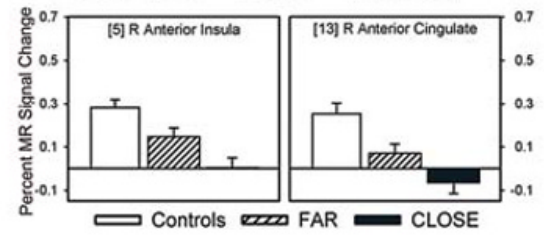

FAR $=$ Controls $>$ CLOSE

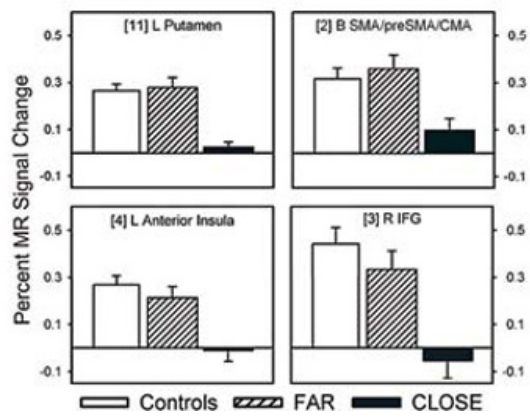

FIGURE 8.5 Regions showing abnormal activation during motor timing in prodromal HD. Top: Map of the functional regions of interest, which were derived from a conjunction map that was used to test for group differences in signal intensity. Green line in the sagittal image is perpendicular to the anterior-posterior commissure. $Z$ coordinate $=m m$ superior to the anterior commissure -posterior commissure line. Bottom: The graphs display the percent MRI signal change for the control, $F A R$, and CLOSE groups. Three different patterns of activation were uncovered: 1) $F A R>$ Controls = CLOSE; 2) Control $>$ FAR $>$ CLOSE; and 3) FAR = Controls $>$ CLOSE. $B=$ bilateral hemispheres; $L=$ left hemisphere; $R=$ right hemisphere. Figure adapted from Zimbelman and colleagues (2007). 
fMRI variables as predictors. Classification accuracy was $60.7 \%$ for caudate and putamen volumes; the cognitive and timing measures did not add to the classification accuracy. However, M RI signal intensity in the left medial frontal gyrus, right cerebellum, and right anterior insula increased classification accuracy to $86.8 \%(83.3,84.6$, and $92.3 \%$ for the control, FAR, and CLOSE groups, respectively). Recall that in the FAR group two of these regions showed hyperactivity (left medial frontal gyrus and right cerebellum), whereas the right anterior insula was hypoactive. Thus, the addition of these variables improved classification the most for the FAR group (46.2\% without fMRI; $84.6 \%$ with fMRI). Though hyperactivity in motor regions may relate to early compensatory mechanisms, the insula is associated with timing-related activation in young adults (Harrington et al., 2010; Kosillo and Smith, 2010; Wittmann et al., 2010a,b) and mediates the synthesis of temporal information across the senses (Harrington et al., 2011a). Altogether, our findings indicate that motor timing probes for corticostriatal functioning in key centers that are early markers of disease prognosis.

\subsubsection{Perceptual Timing}

To minimize the potential role of motor output factors on timing, we also conducted an fMRI study of time discrimination in prodromal HD and in healthy controls (Paulsen et al., 2004). Participants judged whether a CI was longer or shorter in duration than a SI (1200ms) as they underwent fMRI. The single intensity associated with this task was compared to a sensorimotor control task, in which participants simply pressed a button after the presentation of two isochronous tone pairs. The fMRI measure of interest was volume of activated tissue within regions commonly associated with time perception, namely the thalamus, the caudate, the putamen, and the preSMA/cingulate motor area. Prodromal HD participants were separated into two groups of 7 individuals each. One group was FAR from estimated diagnosis (more than 12 years) and the other was CLOSE to a manifest diagnosis (less than 12 years). Due to the small sample sizes, direct comparisons between the groups were not conducted for the fMRI analyses. The behavioral results revealed that time discrimination accuracy was reduced in the CLOSE group, but normal in the FAR group relative to the controls. Significant atrophy was also found in the CLOSE, but not the FAR group.

Figure 8.6 displays the spatial extent of activation in the regions of interest for each group, wherein the activated volume was greater for the timing than the control task. Despite normal timing performance and an absence of striatal atrophy, individuals in the FAR group demonstrated a larger spatial extent of activation in the preSMA and the cingulate motor area relative to the 
control group (Figure 8.6, bottom graph). We speculated that this finding may reflect compensation for early striatal dysfunction. Although this result cannot be directly compared to findings from our study of motor timing (Zimbelman et al., 2007), presumed compensatory activation in the FAR group may differ for time perception than for motor timing (sensorimotor areas) due to differences between the timing tasks in the emphasis placed on context-specific processes. In addition, the spatial extent of thalamus and striatal activation was reduced in the FAR group, whereas the spatial extent of activation in all regions of interest was reduced in the CLOSE group. These preliminary results would be strengthened by larger samples of prodromal HD participants and by conducting direct group comparisons on M R signal intensity across all regions that demonstrate timing-related activation. However, they suggest that time perception deficits in prodromal HD are related to dysfunction in elements of the motor circuit, just as they are in PD for motor timing and time perception (Cerasa et al., 2006; Elsinger et al., 2003; Harrington et al., 2011b; Yu et al., 2007).

\section{Clinical Implications and Future Directions}

Knowledge of the neural mechanisms associated with temporal processing disturbances in PD and HD is beginning to inform an understanding of normal timing, wherein converging findings support the view that timing emerges from interactions of the striatum with extensive brain networks that govern cognitive-control and sensory processing (Bueti, 2011; Merchant et al., 2013). There is also mounting evidence that disturbances in timing have clinical significance. In PD, freezing of gait and finger movements benefit from predictable pacing cues (McIntosh et al., 1997; Spildooren et al., 2012; Thaut et al., 1996; Vercruysse et al., 2012). When cues are withdrawn, motor control deteriorates resulting in smaller movement amplitudes, a hastening or more variable frequency of movements, and decreased coordination stability. Isochronous auditory or visual cues also improve gait in PD without freezing (Georgiou et al., 1993; Lee et al., 2012; Majsak et al., 1998; Nieuwboer et al., 2009). The motor circuit normally governs the internal generation of beat processing (Grahn, 2009; Grahn and Rowe, 2009), which is impaired in PD (Grahn and Brett, 2009). One proposal is that the effective utilization of external, predictable temporal cues in PD may be due to bypassing the frontostriatal system in favor of cerebellar-frontoparietal pathways (Kotz and Schwartze, 2011), which also support beat-based timing (Penhune, Zatorre, and Evans, 1998). This explanation may also account for the greater ease in performing externally- guided than self-initiated movements, the latter of which also 


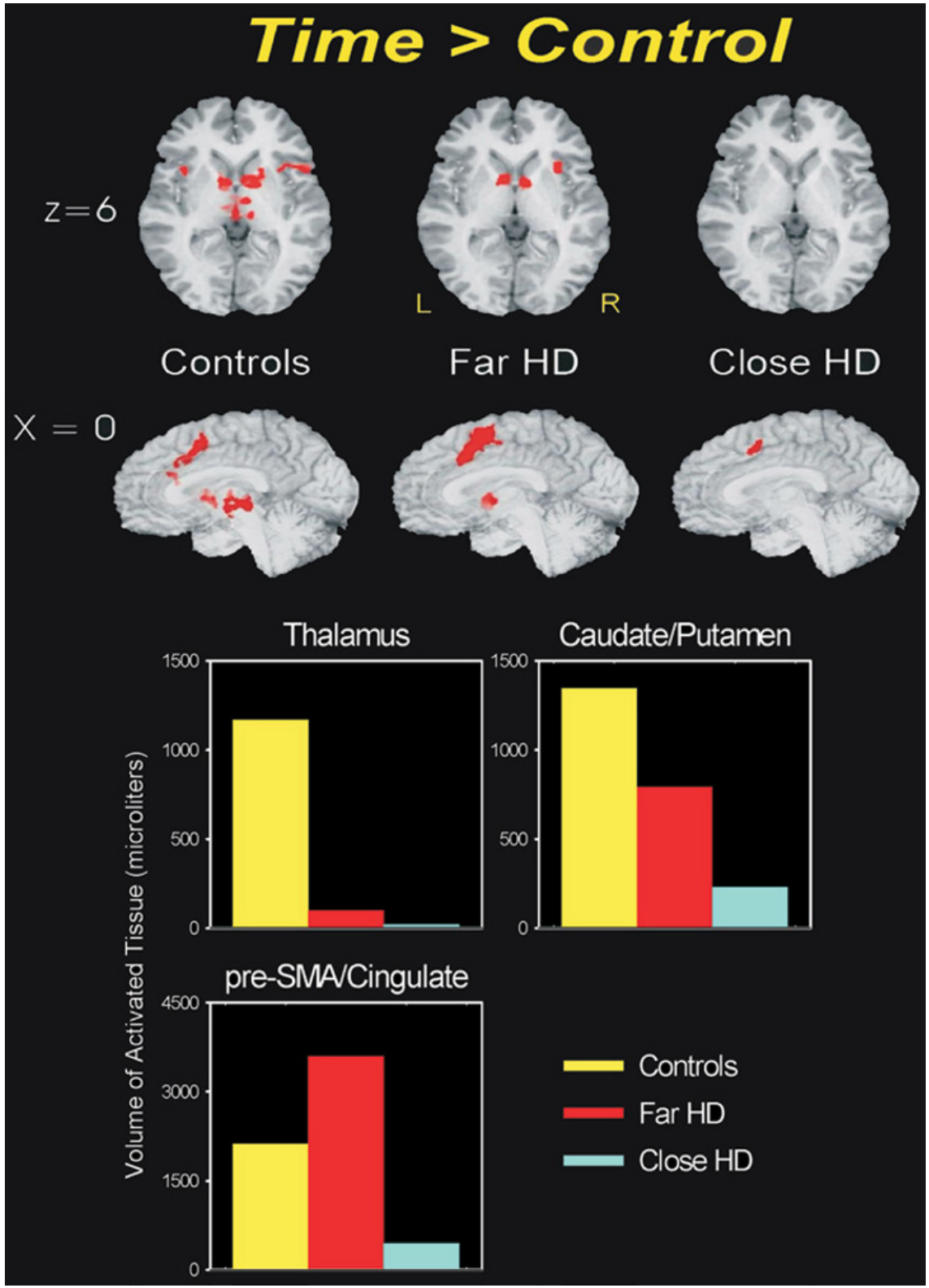

FIGURE 8.6 Regions showing abnormal activation volume during time discrimination in prodromal HD. Top: Activation foci $(p<0.01)$ were derived from the timing minus control task comparison for the control, FAR, and CLOSE groups. L = left hemisphere; $R=$ right hemisphere; $z$ coordinate $=$ mm superior to the anterior commissureposterior commissure line. Bottom: Volume of activation in the thalamus, caudate/ putamen, and the preSMA/cingulate. Figure adapted from Paulsen and colleagues (2004). 
emphasize motor circuit functioning (Cunnington et al., 1995; Jahanshahi et al., 1995; Wu et al., 2011). An alternative explanation is that predictably-timed external cues trigger the execution of a motor plan in premotor cortex, which then suppresses pathological activity in the STN, thereby facilitating movement (Amirnovin et al., 2004; Kuhn et al., 2004). Recent support for this proposal comes from the finding of reduced beta oscillations and increased direction tuning in the STN of individuals with PD when a motor plan was triggered by predictable, but not unpredictable cues (Sarma et al., 2012). Future research is needed to evaluate these hypotheses and to determine whether other compensatory routes to action might also be engaged depending upon the clinical phenotype (e.g., freezing of gait versus no freezing; Snijders et al., 2011). Altogether, this body of work underscores the clinical importance of internal timing disturbances in PD and suggests that timing is closely linked to motor planning disturbances, which are found both in PD and HD (Georgiou et al., 1994, 1995; Harrington and Haaland, 1991; Stout et al., 2011; Wu et al., 2011).

Temporal processing disturbances are also of clinical importance in prodromal HD, where timing ability is a strong marker of disease burden (Rowe et al., 2010; Stout et al., 2011). However, there are also subtle changes in a myriad of other functions including attention, working memory, processing speed, emotion processing, motor speed, and olfaction (Duff et al., 2010; Johnson et al., 2007; Paulsen et al., 2008; Pirogovsky et al., 2007; Say et al., 2011; Stout et al., 2011). With the development of treatments that putatively delay the onset of clinical signs and slow the progression of pathological changes, there has been a concerted effort to determine when changes on different measures can be reliably detected so as to evaluate their potential for outcomes in clinical trials. To this end, we used factor analysis to characterize cognitive domains underlying performances in prodromal HD on a large test battery that included the paced tapping task, and then evaluated their sensitivity in predicting time to diagnosis using a survival analysis (Harrington et al., 2012). Six factors were identified including speed-inhibition, working memory, motor planning, attention-information integration, sensory-perceptual processing, and declarative memory. Almost all tests clearly loaded on one of the six factors, except the paced-tapping task, which loaded on both the motor-planning factor (e.g., choice RT and planning tasks) and the sensory-perceptual factor (e.g., emotion and olfactory processing tasks). This finding was conceptually meaningful since timing proficiency is a facet of planning and perception. Importantly, only the motor-planning and sensory-perceptual factors uniquely predicted time to diagnosis, after controlling for measures of disease burden and motor symptoms. Although the neural basis of these results is unknown, 
tests of motor planning and timing both emphasize processing in frontostriatal circuits (Wu et al., 2011). In contrast, sensory-perceptual processes involved in emotion recognition, olfaction, and timing also probe for functioning in the ventral striatum and limbic systems (e.g., orbitofrontal, cingulate, and anterior insula; Fan et al., 2011; Harrington et al., 2011a; Walla, 2008). The insular cortex and cingulate motor area may be particularly important, as functioning in these regions is abnormal during motor timing in prodromal HD (Zimbelman et al., 2007). Thus, timing proficiency is an important marker of disease prognosis that may prove to be a useful outcome for clinical trials of HD treatments.

A more complete appreciation for the clinical utility of temporal processing measures in neurodegenerative disorders of the basal ganglia will likely come about as functional imaging investigations in PD and prodromal HD develop more fully. Despite current insights into the brain regions that are associated with timing disturbances in these diseases, much remains unknown about potential changes in interactions among brain regions. Regional activation, which is the conventional measure of brain dysfunction, is insensitive to communication among brain regions. Since temporal processing depends on the communication among brain regions, it will be important to study changes in the functional connectivity of brain circuits in diseases. Emerging research suggests that measures of functional connectivity are more sensitive to neurodegeneration (Rowe, 2010) and to the effects of dopamine therapy on timing in PD (Harrington et al., 2011b; Jahanshahi et al., 2010). These measures may also better elucidate potential compensatory routes to timing in basal ganglia disorders (Jahanshahi et al., 2010). In addition, there is a strong need for longitudinal studies that examine the rate of change in timing-related systems to help identify the best biomarkers of early brain-network dysfunction.

In the future, it will also be important to evaluate more complex forms of temporal processing in diseases, since they may be more sensitive to pathology. One area that has received only scant attention in functional imaging studies is intersensory timing. In naturalistic settings, temporal information from different senses is routinely combined into a single perceptual experience. We recently reported that striatum governs intersensory timing through its interactions with the cortex (Harrington et al., 2011a), which concurs with its role in multisensory integration (Nagy et al., 2006) and the integration of cortical oscillatory activity that comprises the clock signal (Matell and Meck, 2004). Another example is research into illusions of time, which are important because they reveal how the brain organizes and construes time depending of internal states (e.g., emotionally charged, high arousal; Dirnberger et al., 2012) or properties of stimuli (Harrington et al., 2011a; Wittmann et al., 2010b). This is 
relevant clinically because distortions in temporal illusions are beginning to elucidate mechanisms for timing in other diseases that alter dopamine system functioning such as schizophrenia (Carroll et al., 2008; Penney et al., 2005). These are merely two examples of many fascinating avenues for future research that are relevant to basal ganglia disorders. With the explosion of research into timing in healthy individuals and animal models in the last decade (Merchant et al., 2013), we are optimistic that this exciting work will soon translate into studies of neurodegenerative disorders.

\section{Acknowledgments}

This chapter was supported by funding from the Department of Veterans Affairs (IIo1CXooo146-01) and the NINDS (NSo40o68). The authors would like to thank Christopher Fong, Jason Reed, and Gabriel Castillo for their technical assistance.

\section{References}

Akkal, D., Dum, R.P., and Strick, P.L. 2007. "Supplementary Motor Area and Presupplementary Motor Area: Targets of Basal Ganglia and Cerebellar Output" JNeurosci, 27, 10659-73.

Allman, M.J. and Meck, W.H. 2012. "Pathophysiological Distortions in Time Perception and Timed Performance" Brain, 135, 656-77.

Amirnovin, R. et al. 2004. "Visually Guided Movements Suppress Subthalamic Oscillations in Parkinson's Disease Patients"J Neurosci, 24, 11302-6.

Artieda, J. et al. 1992. "Temporal Discrimination is Abnormal in Parkinson's Disease" Brain, 115, 199-210.

Aylward, E.H. et al. 2012. "Striatal Volume Contributes to the Prediction of Onset of Huntington Disease in Incident Cases" Biol.Psychiatry, 71, 822-8.

Aylward, E.H. et al. 2011. "Longitudinal Change in Regional Brain Volumes in Prodromal Huntington Disease" J.Neurol.Neurosurg.Psychiatry, 82, 405-10.

Balci, F. et al. 2008. "Pharmacological Manipulations of Interval Timing Using the Peak Procedure in Male $\mathrm{C}_{3} \mathrm{H}$ Mice" Psychopharmacology (Berl), 201, 67-80.

Balci, F. et al. 2012. "Epistasis Effects of Dopamine Genes on Interval Timing and Reward Magnitude in Humans" Neuropsychologia.

Baudrexel, S. et al. 2011. "Resting State fMRI Reveals Increased Subthalamic NucleusMotor Cortex Connectivity in Parkinson's Disease” Neuroimage., 55, 1728-38.

Bechtel, N. et al. 2010. "Tapping Linked to Function and Structure in Premanifest and Symptomatic Huntington Disease" Neurology, 75, 2150-6o. 
Bergman, H. et al. 1998. "Physiological Aspects of Information Processing in the Basal Ganglia of Normal and Parkinsonian Primates" Trends in Neuroscience, 21, 32-8.

Beste, C. et al. 2007. "Time Processing in Huntington's Disease: A Group-Control Study" PLoS.ONE., 2, e1263.

Bostan, A.C., Dum, R.P., and Strick, P.L. 2010. "The Basal Ganglia Communicate with the Cerebellum" Proc.Natl.Acad.Sci.U.S.A., 107, 8452-6.

Brinkman, R.R. et al. 1997. "The Likelihood of Being Affected with Huntington Disease by a Particular Age, for a Specific CAg Size" Am.J.Hum.Genet., 6o, 1202-10.

Bronfeld, M. and Bar-Gad, I. 2011. "Loss of Specificity in Basal Ganglia Related Movement Disorders" Front Syst.Neurosci., 5, 38.

Bueti, D. 2011. "The Sensory Representation of Time" Front Integr.Neurosci., 5, 34.

Bueti, D. and Macaluso, E. 2011. "Physiological Correlates of Subjective Time: Evidence for the Temporal Accumulator Hypothesis" Neuroimage., 57, 1251-63.

Bueti, D., van Dongen, E.V., and Walsh, V. 2008. "The Role of Superior Temporal Cortex in Auditory Timing" PLoS.ONE., 3, e2481.

Buhusi, C.V. and Meck, W.H. 2002. "Differential Effects of Methamphetamine and Haloperidol on the Control of an Internal Clock" Behav.Neurosci., 116, 291-7.

Carbon, M., Edwards, C., and Eidelberg, D. 2003. "Functional Brain Imaging in Parkinson's Disease" Adv.Neurol, 91, 175-81.

Carroll, C.A. et al. 2008. "Temporal Processing Dysfunction in Schizophrenia" Brain and Cognition, 67, 150-61.

Celone, K.A. et al. 2006. "Alterations in Memory Networks in Mild Cognitive Impairment and Alzheimer's Disease: An Independent Component Analysis" J.Neurosci, 26, 10222-31.

Cerasa, A. et al. 2006. "Functional Changes in the Activity of Cerebellum and Frontostriatal Regions during Externally and Internally Timed Movement in Parkinson's Disease" Brain Res Bull., 71, 259-69.

Clower, D.M., Dum, R.P., and Strick, P.L. 2005. "Basal Ganglia and Cerebellar Inputs to 'AIP'” Cereb.Cortex, 15, 913-20.

Cools, R. 2006. "Dopaminergic Modulation of Cognitive Function-Implications for l-DOPA Treatment in Parkinson's Disease" Neurosci Biobehav.Rev., 30, 1-23.

Costa, R.M. et al. 2006. "Rapid Alterations in Corticostriatal Ensemble Coordination during Acute Dopamine-Dependent Motor Dysfunction" Neuron, 52, 359-69.

Coull, J.T., Cheng, R.K., and Meck, W.H. 2011. "Neuroanatomical and Neurochemical Substrates of Timing" Neuropsychopharmacology, 36, 3-25.

Coull, J.T., Nazarian, B., and Vidal, F. 2008. "Timing, Storage, and Comparison of Stimulus Duration Engage Discrete Anatomical Components of a Perceptual Timing Network" J Cogn Neurosci, 20, 2185-97.

Coull, J.T. et al. 2004. "Functional Anatomy of the Attentional Modulation of Time Estimation" Science, 303, 1506-8. 
Cunnington, R. et al. 1995. “Movement-Related Potentials in Parkinson's Disease. Presence and Predictability of Temporal and Spatial Cues" Brain, 118 (Pt 4), 935-50.

Dirnberger, G. et al. 2012. "Give It Time: Neural Evidence for Distorted Time Perception and Enhanced Memory Encoding in Emotional Situations" Neuroimage, 63, 591-9.

Duff, K. et al. 2010. "Mild Cognitive Impairment in Prediagnosed Huntington Disease" Neurology.

Dumas, E.M. et al. 2012. "Early Changes in White Matter Pathways of the Sensorimotor Cortex in Premanifest Huntington's Disease" Hum.Brain Mapp., 33, 203-12.

Dusek, P. et al. 2012. "Abnormal Activity in the Precuneus during Time Perception in Parkinson's Disease: An fMRI Study" PLoS.ONE., 7, e29635.

Elsinger, C.L. et al. 2003. "Neural Basis for Impaired Time Reproduction in Parkinson's Disease: An fMRI Study" J Int.Neuropsychol.Soc., 9, 1088-98.

Fan, J. et al. 2011. "Involvement of the Anterior Cingulate and Frontoinsular Cortices in Rapid Processing of Salient Facial Emotional Information" Neuroimage, 54, 2539-46.

Freeman, J.S. et al. 1996. "Abnormalities of Motor Timing in Huntington's Disease" Parkinsonism and Related Disorders, 2, 81-93.

Gatev, P., Darbin, O., and Wichmann, T. 2006. "Oscillations in the Basal Ganglia under Normal Conditions and in Movement Disorders" Mov Disord., 21, 1566-77.

Georgiou, N. et al. 1993. "An Evaluation of the Role of Internal Cues in the Pathogenesis of Parkinsonian Hypokinesia" Brain, 116 (Pt 6), 1575-87.

Georgiou, N. et al. 1994. "Reduction in External Cues and Movement Sequencing in Parkinson's Disease” J.Neurol.Neurosurg.Psychiatry, 57, 368-70.

Georgiou, N. et al. 1995. "Reliance on Advance Information and Movement Sequencing in Huntington's Disease" Mov Disord., 10, 472-81.

Gibbon, J. 1977. "Scalar Expectancy Theory and Weber's Law in Animal Timing" Psychological Review, 84, 279-325.

Gibbon, J., Church, R.M., and Meck, W.H. 1984. "Scalar Timing in Memory" Annals of the New York Academy of Sciences, 423, 52-77.

Grahn, J.A. 2009. "The Role of the Basal Ganglia in Beat Perception: Neuroimaging and Neuropsychological Investigations" Ann.N.Y.Acad.Sci., 1169, 35-45.

Grahn, J.A. and Brett, M. 2009. "Impairment of Beat-based Rhythm Discrimination in Parkinson's Disease" Cortex, 45, 54-61.

Grahn, J.A. and Rowe, J.B. 20og. "Feeling the Beat: Premotor and Striatal Interactions in Musicians and Nonmusicians during Beat Perception" J.Neurosci., 29, 7540-8.

Hammond, C., Bergman, H., and Brown, P. 2007. "Pathological Synchronization in Parkinson's Disease: Networks, Models and Treatments" Trends Neurosci., 30, 357-64.

Harrington, D.L. and Haaland, K.Y. 1991. "Sequencing in Parkinson's Disease: Abnormalities in Programming and Controlling Movement" Brain, 114, 99-115. 
Harrington, D.L., Haaland, K.Y., and Hermanowicz, N. 1998. "Temporal Processing in the Basal Ganglia" Neuropsychology, 12, 3-12.

Harrington, D.L., Haaland, K.Y., and Knight, R.T. 1998. "Cortical Networks Underlying Mechanisms of Time Perception" JNeurosci, 18, 1085-95.

Harrington, D.L. et al. 2004a. "Does the Representation of Time Depend on the Cerebellum?: Effect of Cerebellar Stroke" Brain, 127, 561-74.

Harrington, D.L. et al. 2004b. "Neural Representation of Interval Encoding and Decision Making" Cognitive Brain Research, 21, 193-205.

Harrington, D.L. et al. 2010. "Neural Modulation of Temporal Encoding, Maintenance, and Decision Processes" Cereb.Cortex, 20, 1274-85.

Harrington, D.L. et al. 2011a. "Neural Underpinnings of Distortions in the Experience of Time across Senses" Front Integr.Neurosci., 5, 32.

Harrington, D.L. et al. 2011b. "Neurobehavioral Mechanisms of Temporal Processing Deficits in Parkinson's Disease" PLoS.ONE., 6, e17461.

Harrington, D.L. et al. 2012. "Cognitive Domains that Predict Time to Diagnosis in Prodromal Huntington Disease" J.Neurol.Neurosurg.Psychiatry, 83, 612-9.

Hinton, S.C. et al. 2007. "Motor Timing Variability Increases in Preclinical Huntington's Disease Patients as Estimated Onset of Motor Symptoms Approaches" J.Int. Neuropsychol.Soc., 13, 539-43.

Hohn, S. et al. 2011. "Behavioral and in Vivo Electrophysiological Evidence for Presymptomatic Alteration of Prefrontostriatal Processing in the Transgenic Rat Model for Huntington Disease" J.Neurosci., 31, 8986-97.

Hoshi, E. et al. 2005. "The Cerebellum Communicates with the Basal Ganglia" Nat. Neurosci, 8, 1491-3.

Ivry, R.B. and Keele, S.W. 1989. "Timing Functions of the Cerebellum" J Cogn Neurosci, $1,136-52$.

Jahanshahi, M. et al. 1995. "Self-initiated Versus Externally Triggered Movements: 1. An Investigation Using Measurement of Regional Cerebral Blood Flow with PET and Movement-Related Potentials in Normal and Parkinson's Disease Subjects" Brain, $118,913-33$.

Jahanshahi, M. et al. 2010. "Dopaminergic Modulation of Striato-Frontal Connectivity during Motor Timing in Parkinson's Disease” Brain, 133, 727-45.

Johnson, S.A. et al. 2007. "Beyond Disgust: Impaired Recognition of Negative Emotions Prior to Diagnosis in Huntington's Disease” Brain, 130, 1732-44.

Jones, C.R. et al. 2008. "Basal Ganglia, Dopamine and Temporal Processing: Performance on Three Timing Tasks on and off Medication in Parkinson's Disease” Brain and Cognition.

Jones, C.R. et al. 2011. "Modeling Accuracy and Variability of Motor Timing in Treated and Untreated Parkinson's Disease and Healthy Controls" Front Integr.Neurosci., 5, 81. 
Koch, G., Oliveri, M., and Caltagirone, C. 2009. "Neural Networks Engaged in Milliseconds and Seconds Time Processing: Evidence from Transcranial Magnetic Stimulation and Patients with Cortical or Subcortical Dysfunction" Philos. Trans.R.Soc.Lond B Biol.Sci., 364, 1907-18.

Koch, G. et al. 2004a. "Subthalamic Deep Brain Stimulation Improves Time Perception in Parkinson's Disease" NeuroReport, 15, 1071-3.

Koch, G. et al. 2004b "High-Frequency rTMS Improves Time Perception in Parkinson Disease" Neurology, 63, 2405-6.

Koch, G. et al. 2008. "Impaired Reproduction of Second but Not Millisecond Time Intervals in Parkinson's Disease" Neuropsychologia, 46, 1305-13.

Kosillo, P. and Smith, A.T. 2010. "The Role of the Human Anterior Insular Cortex in Time Processing” Brain Struct.Funct., 214, 623-8.

Kotz, S.A. and Schwartze, M. 2011. "Differential Input of the Supplementary Motor Area to a Dedicated Temporal Processing Network: Functional and Clinical Implications" Front Integr.Neurosci., 5, 86.

Kuhn, A.A. et al. 2004. "Event-Related Beta Desynchronization in Human Subthalamic Nucleus Correlates with Motor Performance" Brain, 127, 735-46.

Kwak, Y. et al. 2010. "Altered Resting State Cortico-Striatal Connectivity in Mild to Moderate Stage Parkinson's Disease" Front Syst.Neurosci., 4, 143.

Lake, J.I. and Meck, W.H. 2012. "Differential Effects of Amphetamine and Haloperidol on Temporal Reproduction: Dopaminergic Regulation of Attention and Clock Speed" Neuropsychologia.

Lee, S.J. et al. 2012. "The Effects of Visual and Auditory Cues on Freezing of Gait in Patients with Parkinson Disease" Am.J.Phys.Med.Rehabil., 91, 2-11.

Lewis, P.A. and Miall, R.C. 2003. "Brain Activation Patterns during Measurement of Sub- and Supra-second Intervals" Neuropsychologia, 41, 1583-92.

Lewis, S.J. et al. 2003. "Using Executive Heterogeneity to Explore the Nature of Working Memory Deficits in Parkinson's Disease" Neuropsychologia, 41, 645-54.

Lustig, C., Matell, M.S., and Meck, W.H. 2005. "Not 'Just' a Coincidence: Frontal-Striatal Interactions in Working Memory and Interval Timing" Memory, 13, 441-8.

Majsak, M.J. et al. 1998. “The Reaching Movements of Patients with Parkinson's Disease under Self-determined Maximal Speed and Visually Cued Conditions" Brain, 121 (Pt 4), 755-66.

Malapani, C., Deweer, B., and Gibbon, J. 2002. "Separating Storage from Retrieval Dysfunction of Temporal Memory in Parkinson's Disease" JCogn Neurosci, 14, 311-22. Maricq, A.V. and Church, R.M. 1983. "The Differential Effects of Haloperidol and Methamphetamine on Time Estimation in the Rat" Psychopharmacology, 79, 10-5.

Matell, M.S. and Meck, W.H. 2004. "Cortico-Striatal Circuits and Interval Timing: Coincidence Detection of Oscillatory Processes" Cognitive Brain Research, 21, 139-70. 
Matell, M.S., Meck, W.H., and Nicolelis, M.A. 2003. "Interval Timing and the Encoding of Signal Duration by Ensembles of Cortical and Striatal Neurons" Behavioral Neurosciences, $117,760-73$.

Matell, M.S. et al. 2011. "A Heterogeneous Population Code for Elapsed Time in Rat Medial Agranular Cortex" Behav.Neurosci., 125, 54-73.

McIntosh, G.C. et al. 1997. "Rhythmic Auditory-Motor Facilitation of Gait Patterns in Patients with Parkinson's Disease" J Neurol Neurosurg.Psychiatry, 62, 22-6.

Meck, W.H. 1996. "Neuropharmacology of Timing and Time Perception" Cognitive Brain Research, 3, 227-42.

Meck, W.H. 2006a. "Frontal Cortex Lesions Eliminate the Clock Speed Effect of Dopaminergic Drugs on Interval Timing" Brain Res., 1108, 157-67.

Meck, W.H. 2006b. "Neuroanatomical Localization of an Internal Clock: A Functional Link between Mesolimbic, Nigrostriatal, and Mesocortical Dopaminergic Systems" Brain Res, 1109, 93-107.

Meck, W.H. et al. 2012. "Gene-Dose Dependent Effects of Methamphetamine on Interval Timing in Dopamine-Transporter Knockout Mice" Neuropharmacology, 62, 1221-29.

Melgire, M. et al. 2005. "Auditory/visual Duration Bisection in Patients with Left or Right Medial-Temporal Lobe Resection" Brain and Cognition, 58, 119-24.

Merchant, H., Harrington, D.L., and Meck, W.H. 2013. "Neural Basis of the Perception and Estimation of Time" Annual Review of Neuroscience, 36 .

Merchant, H. et al. 2008. "Interval Timing and Parkinson's Disease: Heterogeneity in Temporal Performance" Exp.Brain Res, 184, 233-48.

Nagy, A. et al. 2006. "Multisensory Integration in the Basal Ganglia" Eur.J Neurosci, 24, 917-24.

Nieuwboer, A. et al. 2009. "Upper Limb Movement Interruptions are Correlated to Freezing of Gait in Parkinson's Disease” Eur.J.Neurosci., 29, 1422-30.

Nopoulos, P.C. et al. 2010. "Cerebral Cortex Structure in Prodromal Huntington Disease" Neurobiol.Dis., 40, 544-54.

O'Boyle, D.J., Freeman, J.S., and Cody, F.W.J. 1996. "The Accuracy and Precision of Timing of Self-paced, Repetitive Movements in Subjects with Parkinson's Disease" Brain, 119, 51-70.

Pastor, M.A. et al. 1992a. "Performance of Repetitive Wrist Movements in Parkinson's Disease" Brain, 115, 875-91.

Pastor, M.A. et al.1992b. “Time Estimation and Reproduction is Abnormal in Parkinson's Disease" Brain, 115, 225.

Paulsen, J.S. et al. 2004. "fMRI Biomarker of Early Neuronal Dysfunction in Presymptomatic Huntington's Disease” AJNR Am.J Neuroradiol., 25, 1715-21.

Paulsen, J.S. et al. 2008. "Detection of Huntington's Disease Decades before Diagnosis: The Predict-HD Study" JNeurol Neurosurg.Psychiatry, 79, 874-80. 
Paulsen, J.S. et al. 2010. "Striatal and White Matter Predictors of Estimated Diagnosis for Huntington Disease" Brain Res.Bull., 82, 201-7.

Penhune, V.B., Zatorre, R.J., and Evans, A. 1998. "Cerebellar Contributions to Motor Timing: A PET Study of Auditory and Visual Rhythm Reproduction" JCogn Neurosci, 10, $75^{2-65}$.

Penney, T.B. et al. 2005. "Interval-Timing Deficits in Individuals at High Risk for Schizophrenia" Brain and Cognition, 58, 109-18.

Perbal, S. et al. 2005. "Effects of Internal Clock and Memory Disorders on Duration Reproductions and Duration Productions in Patients with Parkinson's Disease" Brain and Cognition, 58, 35-48.

Pessiglione, M. et al. 2005. “An Effect of Dopamine Depletion on Decision-making: The Temporal Coupling of Deliberation and Execution" J Cogn Neurosci, 17, 1886-96.

Pirogovsky, E. et al. 2007. "Impairments in Source Memory for Olfactory and Visual Stimuli in Preclinical and Clinical Stages of Huntington's Disease" J Clin.Exp. Neuropsychol., 29, 395-404.

Pouthas, V. et al. 2005. "Neural Network Involved in Time Perception: An fMRI Study Comparing Long and Short Interval Estimation" Human Brain Mapping, 25, 433-41.

Rammsayer, T.H. 1993. "On Dopaminergic Modulation of Temporal Information Processing" Biol.Psychol., 36, 209-22.

Rammsayer, T. and Classen, W. 1997. "Impaired Temporal Discrimination in Parkinson's Disease: Temporal Processing of Brief Durations as an Indicator of Degeneration of Dopaminergic Neurons in the Basal Ganglia" Int.J.Neurosci., 91, 45-55.

Ranen, N.G. et al. 1995. "Anticipation and Instability of IT-15 (CAG)n Repeats in ParentOffspring Pairs with Huntington Disease" Am.J.Hum.Genet., 57, 593-602.

Rao, S.M. et al. 1997. "Distributed Neural Systems Underlying the Timing of Movements" JNeurosci, $17,55^{28-35}$.

Rao, S.M., Mayer, A.R., and Harrington, D.L. 2001. "The Evolution of Brain Activation during Temporal Processing" Nature Neuroscience, 4, 317-23.

Riesen, J.M. and Schnider, A. 2001. "Time Estimation in Parkinson's Disease: Normal Long Duration Estimation Despite Impaired Short Duration Discrimination" J.Neurol., 248, 27-35.

Rowe, J.B. 2010. "Connectivity Analysis is Essential to Understand Neurological Disorders" Front Syst.Neurosci., 4.

Rowe, K.C. et al. 2010. "Self-paced Timing Detects and Tracks Change in Prodromal Huntington Disease" Neuropsychology, 24, 435-42.

Sarma, S.V. et al. 2012. "The Effects of Cues on Neurons in the Basal Ganglia in Parkinson's Disease" Front Integr.Neurosci., 6, 40.

Say, M.J. et al. 2011. "Visuomotor Integration Deficits Precede Clinical Onset in Huntington's Disease" Neuropsychologia, 49, 264-70. 
Schwartze, M. et al. 2011. "The Impact of Basal Ganglia Lesions on Sensorimotor Synchronization, Spontaneous MotorTempo, and the Detection of Tempo Changes" Behav.Brain Res., 216, 685-91.

Smith, J.G. et al. 2007. "The Effect of Parkinson's Disease on Time Estimation as a Function of Stimulus Duration Range and Modality" Brain and Cognition, 64, 130-43.

Snijders, A.H. et al. 2011. "Gait-Related Cerebral Alterations in Patients with Parkinson's Disease with Freezing of Gait" Brain, 134, 59-72.

Spencer, R.M. and Ivry, R.B. 2005. "Comparison of Patients with Parkinson's Disease or Cerebellar Lesions in the Production of Periodic Movements Involving Event-based or Emergent Timing" Brain and Cognition, 58, 84-93.

Spildooren, J. et al. 2012. “Turning and Unilateral Cueing in Parkinson's Disease Patients with and without Freezing of Gait" Neuroscience, 207, 298-306.

Stoffers, D. et al. 2008. "Increased Cortico-Cortical Functional Connectivity in EarlyStage Parkinson's Disease: An MEg study" Neuroimage, 41, 212-22.

Stout, J.C. et al. 2011. "Neurocognitive Signs in Prodromal Huntington Disease" Neuropsychology, 25, 1-14.

Taniwaki, T. et al. 2003. "Reappraisal of the Motor Role of Basal Ganglia: A Functional Magnetic Resonance Image Study” J.Neurosci., 23, 3432-38.

Thaut, M.H. et al. 1996. "Rhythmic Auditory Stimulation in Gait Training for Parkinson's Disease Patients" Mov Disord., 11, 193-200.

Thompson, J.C. et al. 2010. "Automaticity and Attention in Huntington's Disease: When Two Hands are Not Better Than One" Neuropsychologia, 48, 171-8.

Tobin, A.J. and Signer, E.R. 200o. "Huntington's Disease: The Challenge for Cell Biologists" Trends Cell Biol., 10, 531-6.

Vercruysse, S. et al. 2012. "Abnormalities and Cue Dependence of Rhythmical UpperLimb Movements in Parkinson Patients with Freezing of Gait" Neurorehabil.Neural Repair, 26, 636-45.

Walla, P. 2008. "Olfaction and Its Dynamic Influence on Word and Face Processing: Cross-Modal Integration" Prog.Neurobiol., 84, 192-209.

Wearden, A.J. 1999. “Beyond the Fields We Know...': Exploring and Developing Scalar Timing Theory" Behavioural Processes, 45, 3-21.

Wearden, J.H. et al. 2008. "Stimulus Timing by People with Parkinson's Disease" Brain and Cognition, 67, 264-79.

Wiener, M. et al. 2010. "Fast Forward: Supramarginal Gyrus Stimulation Alters Time Measurement" J.Cogn Neurosci., 22, 23-31.

Wiener, M. et al. 2012. "Parietal Influence on Temporal Encoding Indexed by Simultaneous Transcranial Magnetic Stimulation and Electroencephalography" J.Neurosci., 32, 12258-67. 
Wild-Wall, N. et al. 2008. "Time Estimation in Healthy Ageing and Neurodegenerative Basal Ganglia Disorders" Neurosci Lett.

Williams-Gray, C.H. et al. 2009. "The Distinct Cognitive Syndromes of Parkinson's Disease: 5 year Follow-up of the CamPaIGN Cohort" Brain, 132, 2958-69.

Wing, A.M., Keele, S.W., and Margolin, D.I. 1984. "Motor Disorder and the Timing of Repetitive Movements" In J.Gibbon and L. Allen (Eds.), Ann NY Acad Sci: Timing Time Perc (pp. 183-92).

Wittmann, M. et al. 2010a. "Accumulation of Neural Activity in the Posterior Insula Encodes the Passage of Time" Neuropsychologia, 48, 3110-20.

Wittmann, M. et al. 2010b. "The Neural Substrates of Subjective Time Dilation" Front Hum.Neurosci., 4, 2.

Wu, T. and Hallett, M. 2005. "A Functional MrI Study of Automatic Movements in Patients with Parkinson's Disease" Brain, 128, 2250-9.

Wu, T., Chan, P., and Hallett, M. 2010. "Effective Connectivity of Neural Networks in Automatic Movements in Parkinson's Disease" Neuroimage, 49, 2581-7.

Wu, T. et al. 2010. "Neural Correlates of Bimanual Anti-phase and In-phase Movements in Parkinson's Disease" Brain, 133, 2394-409.

$\mathrm{Wu}$, T. et al. 2011. "Effective Connectivity of Brain Networks during Self-initiated Movement in Parkinson's Disease" Neuroimage., 55, 204-15.

$\mathrm{Yu}, \mathrm{H}$. et al. 2007. "Role of Hyperactive Cerebellum and Motor Cortex in Parkinson's Disease" Neuroimage, 35, 222-33.

Zimbelman, J.L. et al. 2007. "fMRI Detection of Early Neural Dysfunction in Preclinical Huntington's Disease" J Int.Neuropsychol.Soc., 13, 758-69. 